\title{
Relación entre burnout y empatía en estudiantes de medicina de una universidad privada del Perú
}

\section{Relationship between burnout and empathy in medical students from a private university in Peru}

\author{
Jeff Huarcaya-Victoria, ${ }^{1,2,3, a}$, Gabriel Delfín ${ }^{4, b}$, Diego Huamán ${ }^{4, c}$ \\ ${ }^{1}$ Centro de Investigación en Salud Pública, Facultad de Medicina, Universidad San Martín de Porres. Lima, Perú. \\ ${ }^{2}$ Departamento Académico de Psiquiatria,Facultad de Medicina, Universidad Nacional Mayor de San Marcos. Lima, Perú. \\ ${ }^{3}$ Departamento Académico de Psiquiatría, Hospital Nacional Guillermo Almenara Irigoyen, Seguro Social de Salud EsSalud. Lima, Perú. \\ ${ }^{4}$ Sociedad Cientifica de Estudiantes de Medicina de la Universidad de San Martin de Porres. Lima, Perú. \\ ${ }^{a}$ Médico psiquiatra, ORCID: https://orcid.org/0000-0003-4525-9545 \\ ${ }^{\mathrm{b}}$ Estudiante de medicina, ORCID: https://orcid.org/0000-0002-0736-3141 \\ 'Estudiante de medicina, ORCID: https://orcid.org/0000-0002-8907-9418
}

An Fac med. 2019;80(4):488-93 / DOI: https://doi.org/10.15381/anales.v80i4.16259

\section{Correspondencia: \\ Jeff David Huarcaya Victoria \\ jhuarcayav@usmp.pe}

Recibido: 10 de junio 2019

Aceptado: 20 de noviembre 2019

Publicación en línea: 28 de diciembre 2019

Conflictos de interés: Los autores declaran no tener conflictos de interés.

Fuente de financiamiento:

Autofinanciado

Citar como: Huarcaya-Victoria J, Delfín G, Huamán D. Relación entre burnout y empatía en estudiantes de medicina de una universidad privada del Perú. An Fac med. 2019;80(4):488-93. DOI: https://doi.org/10.15381/anales. v80i4.16259

\section{Resumen}

Introducción. El burnout entre los estudiantes de medicina es una entidad seria y común. Se ha documentado que el burnout se encuentra relacionado con una reducción de la empatía. Objetivo. Evaluar la relación entre el diagnostico de síndrome de burnout y los niveles de empatía en estudiantes de medicina. Métodos. Estudio transversal realizado en 245 estudiantes de medicina humana del primer año, en Lima. Se utilizó la Maslach burnout Inventory-Student Survey (MBI-SS), la Jefferson Scale of Physician Empathy (JSPE-S) y una ficha de recolección de datos sociodemográficos. Resultados. Se encontró una correlación entre el total de la MBI-SS y la JSPE-S ( $p=-0,269 ; p<0,01)$. Las correlaciones entre las dimensiones del burnout y el total de la JSPE-S fueron: agotamiento emocional (AE) ( $p=-0,137 ; p<0,05)$, cinismo $(C I)(p=-0,241 ; p<0,01)$ y eficacia académica $(E A)(p=0,262 ; p<0,01)$. Conclusión. La empatía en estudiantes de medicina muestra una correlación con todas las dimensiones del sindrome de Burnout; de forma negativa con el AE y $\mathrm{Cl}$, y de forma positiva con la EA.

Palabras clave: Agotamiento Psicológico; Empatía; Estudiantes de Medicina; Perú (fuente: DeCS BIREME).

\section{Abstract}

Introduction. Burnout among medical students is a serious and common entity. It has been documented that burnout is related to a reduction in empathy. Objective. To evaluate the relationship between Burnout syndrome and empathy levels in medical students. Methods. A cross-sectional study developed in 245 firstyear medical students in a medical school from Lima. The Maslach Burnout Inventory-Student Survey (MBISS), the Jefferson Scale of Physician Empathy (JSPE-S) and a sociodemographic data collection sheet were used. Results. It was found that a correlation between the total MBI-SS and the JSPE-S ( $p=-0,269 ; p<0,01)$. The correlations between the dimensions of the burnout and the total of the JSPE-S were: emotional exhaustion $(A E)(p=-0,137 ; p<0,05)$, cynicism (Cl) $(p=-0,241 ; p<0,01)$ and academic effectiveness $(E A)(p=0,262$; $p<0,01)$. Conclusions. Empathy in medical students shows a correlation with all the dimensions of burnout; negatively with the $\mathrm{AE}$ and $\mathrm{Cl}$, and positively with the $\mathrm{EA}$.

Keywords: Burnout, Psychological; Empathy; Students, Medical; Peru (source: MeSH NLM). 


\section{INTRODUCCIÓN}

La relación médico-paciente ha sufrido cambios en los últimos años debido al incremento del acceso a la información por parte del paciente y un mayor reconocimiento de sus derechos, lo cual ha fortalecido su autonomía ${ }^{(1)}$. Estos hechos hacen que, en el entorno médico actual, se requiera un cambio en los roles desempeñados por los médicos y el tipo de educación que se brinda a los estudiantes de medicina, siendo necesario aumentar la conciencia sobre el impacto de las actitudes de los médicos en los pacientes y de fomentar la adquisición de las habilidades y estrategias necesarias para lograr una adecuada comunicación ${ }^{(1,2)}$.

De todas estas habilidades, la empatía ha recibido un especial interés. Esta ha sido definida como nuestra capacidad de identificar lo que otra persona siente o piensa y poder responder ante esto con una emoción adecuada, para lo cual es necesario suspender nuestro enfoque de atención único, centrado exclusivamente en nuestra mente, y adoptar un enfoque de atención doble que también se centre en la mente del otro ${ }^{(3)}$.

En la última década, una creciente literatura sobre la empatía en medicina indica que ayudaría a mejorar muchos aspectos de la práctica de la atención médica y la satisfacción del paciente ${ }^{(4,5)}$. Por lo tanto, la empatía es un componente importante para convertirse en un médico profesionalmente competente ${ }^{(6)}$. Al ser de naturaleza multidimensional, la empatía tiene tanto componentes estables como variables, siendo los últimos los que podrían verse afectados por el ambiente, como, por ejemplo, en situaciones estresantes. En este contexto cobra especial interés el síndrome de burnout.

El fenómeno del burnout emergió a mediados de la década de 1970 como un problema social mayor en EE.UU. ${ }^{(7)}$. Se sabe que es de presentación común en estudiantes de medicina (44\%) ${ }^{(8)}$, el cual puede deberse a una mala adaptación, conflictos interpersonales entre estudiantes y profesores, exposición a la muerte y el sufrimiento, factores curriculares, alta carga académica, entre otros ${ }^{(9)}$. Estudios en diversos países han demostrado una relación negativa entre empatía y burnout en estudiantes de medicina $(2,6,8,9)$; sin embargo, aún no se comprende la naturaleza de esta relación ${ }^{(10)}$. Como la empatía es un factor clave en la carrera de un médico, los recursos adecuados de detección y tratamiento de los factores que puedan disminuirla, como el burnout, pueden ser invaluables para los estudiantes de medicina ${ }^{(6)}$.

En el Perú sólo existe una investigación que evalúa la relación entre empatía y burnout en un grupo de internos de ciencias de la salud de un hospital general, en el cual se concluyó que existe una asociación significativa entre ambos (OR: 4,13; IC 95\%: 1,61-10,71) (11). No se ha investigado la relación entre la empatía y el burnout en estudiantes de medicina peruanos, y sobre todo en quienes recién inician su formación médica, siendo esto alarmante, pues no permite que tengamos datos fidedignos que permitan desarrollar posibles estrategias de intervención en etapas tempranas de la formación médica.

El presente estudio se desarrolló con el objetivo de evaluar la relación entre el diagnostico de síndrome de burnout y los niveles de empatía en un grupo de estudiantes de medicina que recién inician su formación de una universidad privada del Perú.

\section{MÉTODOS}

\section{Diseño del estudio}

Se realizó un estudio observacional, transversal realizado en la Facultad de Medicina de la Universidad de San Martín de Porres (FMH-USMP), que buscó evaluar la relación entre los niveles de empatía y burnout en un grupo de estudiantes de medicina.

\section{Población y muestra}

Participaron del estudio todos los estudiantes de medicina de la FMH-USMP del primer año $(n=334)$. La recolección de los datos, la cual estuvo a cargo de los investigadores $\mathrm{GD}$ y $\mathrm{DH}$, se realizó al inicio del semestre académico 2018-II. Como se trabajó con toda la población no fue necesario realizar técnicas de muestreo.

\section{Variables}

La empatía médica fue medida mediante la Jefferson Scale of Physician Empathy en su versión para estudiantes de medicina (JSPE-S). Este instrumento consta de 20 ítems valorados sobre una escala Likert de siete puntos (rango posible de 20 a 140). Valora tres dimensiones: "toma de perspectiva" - TP (diez ítems), "ponerse en los zapatos del paciente" - PP (tres ítems), y "cuidado con compasión" - CC (siete ítems). Esta escala es ampliamente utilizada por investigadores a nivel mundial ${ }^{(12,13)}$, se encuentra validada al castellano, y tiene una consistencia interna de rango aceptable (alfa de Cronbach $=0,74)^{(14)}$. AsImismo, esta escala ya ha sido usada previamente en Perú en muestras de estudiantes de medicina y médicos residentes ${ }^{(15,16)}$.

La evaluación del burnout se realizó utilizando la Maslach Burnout InventoryStudent Survey (MBI-SS). Esta escala fue desarrollada asumiendo que la actividad académica del estudiante podría generar síntomas de agotamiento, cinismo y autoeficacia negativa como se ve en algunos trabajadores ${ }^{(17)}$. Consta de 15 ítems valorados sobre una escala Likert de siete puntos. Valora tres dimensiones: "agotamiento emocional" (AE) (bajo = 0-9; moderado = 10-14; alto > 14), "cinismo" (CI) (bajo = 0-1; moderado = 2-6; alto > 6), y "eficacia académica" (EA) (bajo $\leq 22$; moderado = 23-27; alto $\geq 28)^{(8)}$. Para el diagnóstico del síndrome de burnout se utilizó el criterio de dos dimensiones (altos niveles de $\mathrm{AE}$ con altos niveles de $\mathrm{Cl}$ o bajos niveles de EA) y el de tres dimensiones (altos niveles de $\mathrm{AE}$ y $\mathrm{Cl}$ con bajos niveles de EA) ${ }^{(8)}$. La MBI-SS se encuentra validada al castellano, y tiene una consistencia interna aceptable con unos valores de alfa de Cronbach de 0,77 (AE), 0,72 (Cl), y $0,82(E A)^{(18)}$.

Además de la JSPE-S y la MBI-SS, se entregó una ficha de recolección de datos sociodemográficos, en la que se obtuvo datos sobre la edad, el género, si se tiene pareja actual, lugar de procedencia, religión, haber reprobado un curso y la especialidad que se desea seguir. 


\section{Análisis estadístico}

Se realizaron técnicas de estadística descriptiva para todas las variables. Se evaluó la JSPE-S y la MBI-SS en sus dimensiones por separado mediante sus estadísticos resumen y su distribución. La relación entre la JSPE-S y la MBI-SS con las variables cualitativas se evaluó mediante la prueba de U de Mann-Whitney. Se evaluó la correlación lineal entre los resultados totales y las dimensiones de la JSPE-S y la MBI-SS mediante la Rho de Spearman. El nivel alfa se determinó antes del análisis en 0,05. Los análisis se realizaron con ayuda del programa estadístico IBM SPSS Statistics for Windows, Version 23.0 (IBM Corp. Released $2015^{\circledR}$ ).

\section{Aspectos éticos}

Se siguieron los lineamientos de la Declaración de Helsinki. Se contó además con la autorización de las autoridades y la aprobación del Comité de Ética de la FMH-USMP.

\section{RESULTADOS}

De un total de 334 estudiantes de primer año, 245 contestaron las escalas (tasa de respuesta $=73,3 \%$ ). La edad promedio fue de 17,5 \pm 1,2 años, la edad mínima fue de 16 y la máxima de 24 años (Tabla 1).

Dentro del análisis comparativo según las variables estudiadas para las puntuaciones de JSPE-S, se encontró que la única diferencia estadísticamente significativa ocurrió en los puntajes totales de la JSPE-S $(p=0,007)$ y la subescala "toma de perspectiva" $(p=0,007)$ en la variable pareja (Tabla 2). Encontramos que la JSPE-S tuvo una consistencia interna aceptable (alfa de cronbach=0,789). Además, también encontramos una consistencia interna aceptable en la MBI-SS (alfa de cronbach=0,809).

Los puntajes de la MBI-SS fueron categorizados en sus tres dimensiones. Basado en esta categorización, 95 (38,8\%) estudiantes tuvieron alto $A E, 12$ (4,9\%) altos niveles de $\mathrm{Cl}$, y $28(11,4 \%)$ bajos niveles de EA. De acuerdo al criterio de dos dimensiones (alto $\mathrm{AE}$ con alto $\mathrm{Cl}$ o baja EA), 21 (8,57\%) estudiantes tuvieron el síndrome de burnout, mientras que con el criterio de tres dimensiones (alto $A E$ con alto $\mathrm{Cl}$ y baja $\mathrm{EA}), 6(2,44 \%)$. Se encontró diferencias significativas entre los puntajes de EA y el tener pareja actual (Tabla 3).

El total de la MBI-SS tuvo una correlación negativa significativa con el puntaje total de la JSPE-S ( $p=-0,269 ; p<0,01)$. Respecto a las dimensiones de la MBI-SS, se encontró que el $A E$ se correlacionó con el total de la JSPE-S ( $p=-0,137 ; p<0,05)$. $\mathrm{El} \mathrm{Cl}$ se correlacionó con el total de la JSPE-S $(p=-0,241 ; p<0,01)$. La EA se correlacionó con el total de la JSPE-S ( $p=0,262$; $p<0,01$ ) (Tabla 4).

\section{DISCUSIÓN}

En los estudiantes del primer año de medicina participantes del estudio, encontramos una baja prevalencia del síndrome de burnout. En algunos estudios se ha reportado que, en los estudian-

Tabla 1. Características sociodemográficas de 245 estudiantes de medicina de 1er año de la FMH-USMP.

\begin{tabular}{lc} 
Variable & $\mathbf{n}(\%)$ \\
Edad (media $\pm D E)$ & $17,5 \pm 1,2$ \\
\hline
\end{tabular}

Género

\begin{tabular}{cc}
\hline Masculino & $82(33,5)$ \\
\hline Femenino & $163(66,5)$ \\
\hline Pareja actual & \\
\hline Sí & $49(20)$ \\
\hline No & $196(80)$ \\
\hline
\end{tabular}

Lugar de procedencia

\begin{tabular}{lc}
\hline Lima & $184(75,1)$ \\
\hline Provincia & $58(23,7)$ \\
\hline Extranjero & $3(1,2)$ \\
\hline
\end{tabular}

Religión

\begin{tabular}{cc}
\hline Sí & $186(75,9)$ \\
\hline No & $59(24,1)$ \\
\hline
\end{tabular}

\begin{tabular}{cc}
\hline ¿Ha reprobado algún curso? & \\
\hline Sí & $32(13,1)$ \\
\hline No & $213(86,9)$
\end{tabular}

\begin{tabular}{lc}
\hline Especialidad que desea seguir & \\
\hline \multicolumn{1}{c}{ Centrada en el paciente } & $198(80,8)$ \\
\hline \multicolumn{1}{c}{ Centrada en la tecnología } & $39(15,9)$ \\
\hline $\begin{array}{l}\text { Actividad universitaria en horas } \\
\text { (media } \pm \text { DE) }\end{array}$ & $5,8 \pm 3,1$ \\
\hline $\begin{array}{l}\text { Actividades recreativas en horas } \\
\text { (media } \pm \text { DE) }\end{array}$ & $2,4 \pm 1,7$ \\
\hline
\end{tabular}

DE: Desviación estándar tes que recién inician su formación médica, el burnout clínico es poco prevalente. Por ejemplo, en un estudio realizado en EE.UU., se encontró que los estudiantes durante sus años clínicos tuvieron más probabilidades de experimentar el síndrome de burnout en comparación con aquellos que recién iniciaban su formación médica ${ }^{(19) ;}$ mientras que en otro, también realizado en EE.UU., se halló que el síndrome de burnout estuvo presente en el $71 \%$ de estudiantes de medicina de tercer año, siendo esta cifra mucho mayor a lo reportado en estudiantes de 1er año ${ }^{(20)}$. Se han reportado resultados diferentes, como un estudio en Brasil, en el cual los estudiantes de medicina obtuvieron un alto nivel de burnout desde el 1 er año y en las etapas previas al ingreso a la facultad de medicina, ya que muchos de ellos pueden pasar varios años tratando de ser admitidos en la facultad sin éxito, lo que incrementa considerablemente los niveles de estrés ${ }^{(8)}$.

A pesar de la baja prevalencia del síndrome de burnout en nuestra muestra, encontramos altos niveles de una de sus dimensiones, el AE. Según estudios previos la prevalencia de AE fue del 25,5 - 55,0\%, despersonalización o $\mathrm{Cl}$ en 7,7 - 38,0\%, y disminución de la satisfacción personal o EA en $31,0-46,6 \%{ }^{(21-23)}$. Se sabe que las dimensiones del síndrome de burnout se manifiestan secuencialmente, desarrollándose primero el $\mathrm{AE}$, seguido de la despersonalización o $\mathrm{Cl}$ como una medida de poder lidiar con el AE, y finalmente la capacidad de resistir las demandas del trabajo o estudio disminuyen, lo que resultaría en síntomas afectivos y baja productividad profesional o académica ${ }^{(8)}$. Por lo tanto, es factible suponer que estos altos niveles de AE son esperables en los primeros años de estudio, ya que es la primera dimensión en verse afectada. Un hallazgo común en la literatura señala que las estudiantes mujeres tienden a mostrar mayores niveles de $A E$, mientras que los hombres mayores niveles de $\mathrm{Cl}^{(23,24)}$; sin embargo, esto no fue hallado en nuestro estudio. Otro resultado significativo fue la relevancia de tener una pareja en los niveles totales de la JSPE-S y en la dimensión EA de la MBI-SS. En un estudio se encontró que tener una pareja se relacionaba significativamente con mayo- 
Tabla 2. Análisis comparativo según las variables estudiadas, para las puntuaciones de empatia obtenidas de la Jefferson Scale of Physician Empathy (JSPE).

\begin{tabular}{|c|c|c|c|c|c|c|c|c|c|c|c|c|c|}
\hline \multirow{3}{*}{ Variable } & \multirow{3}{*}{ n (\%) } & \multicolumn{12}{|c|}{ JSPE } \\
\hline & & \multicolumn{3}{|c|}{ TP } & \multicolumn{3}{|c|}{ CC } & \multicolumn{3}{|c|}{ PP } & \multicolumn{3}{|c|}{ Total } \\
\hline & & $X \pm \sigma$ & $\begin{array}{c}\text { Me } \\
\text { (IQR) }\end{array}$ & p & $X \pm \sigma$ & $\begin{array}{c}\text { Me } \\
\text { (IQR) }\end{array}$ & p & $X \pm \sigma$ & $\begin{array}{c}\mathrm{Me} \\
\text { (IQR) }\end{array}$ & p & $X \pm \sigma$ & Me (IQR) & $\mathbf{p}$ \\
\hline \multicolumn{14}{|l|}{ Género } \\
\hline Masculino & $82(33,5)$ & $56,65 \pm 8,11$ & $57(12)$ & 0,103 & $38,91 \pm 6,95$ & $40(10)$ & 0,198 & $13,63 \pm 2,97$ & 14(4) & 0,308 & $109,2 \pm 13,28$ & 110(20) & 0,068 \\
\hline Femenino & $163(66,5)$ & $58,18 \pm 7,9$ & 59(11) & & $39,95 \pm 7,13$ & $41(10)$ & & $14,13 \pm 3,18$ & $14(4)$ & & $112,26 \pm 14,26$ & $114(21)$ & \\
\hline \multicolumn{14}{|l|}{ Pareja actual } \\
\hline Sí & $49(20)$ & $60,37 \pm 7,54$ & 63(11) & 0,007 & $40,67 \pm 6,77$ & $43(10)$ & 0,197 & $14,61 \pm 3,3$ & $14(5)$ & 0,188 & $115,65 \pm 14,18$ & $117(16)$ & 0,007 \\
\hline No & $196(80)$ & $56,99 \pm 7,97$ & $58(12)$ & 0,007 & $39,34 \pm 7,14$ & $41(10)$ & 0,197 & $13,8 \pm 3,05$ & $14(4)$ & 0,188 & $110,13 \pm 13,75$ & $112(21)$ & \\
\hline \multicolumn{14}{|l|}{ Lugar de procedencia } \\
\hline Lima & $184(75,1)$ & $57,52 \pm 8,1$ & $58(12)$ & 0,693 & $39,56 \pm 7,28$ & $41(10)$ & 0,801 & $13,83 \pm 3,1$ & $14(4)$ & 0,200 & $110,91 \pm 14,31$ & $113(21)$ & 0,605 \\
\hline Provincia & $58(23,7)$ & $58,10 \pm 7,9$ & $58,5(11)$ & & $39,66 \pm 6,6$ & $41(9)$ & & $14,47 \pm 3,14$ & $15(4)$ & 0,200 & $112,22 \pm 13,36$ & 114(19) & \\
\hline \multicolumn{14}{|l|}{ Religión } \\
\hline Sí & $186(75,9)$ & $57,61 \pm 8,09$ & $58,5(12)$ & 0,974 & $39,55 \pm 7,18$ & $41(10)$ & 0,971 & $14,13 \pm 3,04$ & $14(4)$ & 0,197 & $111,30 \pm 14,05$ & $113(22)$ & 0,993 \\
\hline No & $59(24,1)$ & $57,83 \pm 7,71$ & $58(12)$ & & $39,76 \pm 6,78$ & $41(9)$ & & $13,44 \pm 3,13$ & $14(4)$ & 0,197 & $111,03 \pm 13,9$ & 113(17) & 0,993 \\
\hline \multicolumn{14}{|l|}{ ¿Ha reprobado algún curso? } \\
\hline Sí & $32(13,1)$ & $56,09 \pm 9,16$ & $57,5(17)$ & 0,371 & $40,63 \pm 5,84$ & $41(10)$ & 0,576 & $13,31 \pm 2,38$ & 13(3) & 0,117 & $110,03 \pm 13,56$ & $113,5(24)$ & 0,614 \\
\hline No & $213(86,9)$ & $57,9 \pm 7,79$ & $59(12)$ & & $39,45 \pm 7,24$ & $41(10)$ & & $14,06 \pm 3,2$ & $14(4)$ & 0,117 & $111,41 \pm 14,07$ & $113(20)$ & \\
\hline \multicolumn{14}{|l|}{ Especialidad que desea seguir } \\
\hline Centrada en el paciente & $98(80,8)$ & $58,09 \pm 7,79$ & 59(11) & 0,186 & $39,49 \pm 7,13$ & $41(10)$ & 0,621 & $14,01 \pm 3,09$ & 14(4) & 0,226 & $111,59 \pm 13,87$ & $113,5(20)$ & 0,428 \\
\hline Centrada en la tecnología & $39(15,9)$ & $56,15 \pm 8,53$ & $56(14)$ & & $40,08 \pm 7,26$ & $41(11)$ & & $13,49 \pm 3,36$ & 13(5) & & $109,72 \pm 14,7$ & 110(23) & \\
\hline
\end{tabular}

TP: Toma de perspectiva; CC: Cuidado con compasión; PP: Ponerse en los zapatos del paciente; JSPE-S: Jefferson Scale of Physician Empathy versión para estudiantes de medicina Valores $p$ obtenidos a través de la prueba U de Mann-Whitney

Tabla 3. Análisis comparativo según las variables estudiadas, para las puntuaciones de Burnout obtenidas de la Maslach Burnout Inventory-Student Survey (MBI-SS).

\begin{tabular}{|c|c|c|c|c|c|c|c|c|c|c|c|c|c|}
\hline \multirow{3}{*}{ Variable } & \multirow{3}{*}{$n(\%)$} & \multicolumn{12}{|c|}{ MBI-SS } \\
\hline & & \multicolumn{3}{|c|}{ AE } & \multicolumn{3}{|c|}{ c } & \multicolumn{3}{|c|}{ EA } & \multicolumn{3}{|c|}{ Total } \\
\hline & & $X \pm \sigma$ & $\begin{array}{c}\text { Me } \\
\text { (IQR) }\end{array}$ & p & $X \pm \sigma$ & $\begin{array}{c}\text { Me } \\
\text { (IQR) }\end{array}$ & $\mathbf{p}$ & $X \pm \sigma$ & $\begin{array}{c}\mathrm{Me} \\
\text { (IQR) }\end{array}$ & $\mathbf{p}$ & $x \pm \sigma$ & $\begin{array}{c}\text { Me } \\
\text { (IQR) }\end{array}$ & $\mathbf{p}$ \\
\hline \multicolumn{14}{|l|}{ Género } \\
\hline Masculino & $82(33,5)$ & $14,02 \pm 6,57$ & $13(8)$ & 0,419 & $1,84 \pm 3,31$ & 1(3) & 0,05 & $27,88 \pm 4,98$ & $28(6)$ & 0,732 & $23,99 \pm 10.74$ & $23(14)$ & 0,505 \\
\hline Femenino & $163(66,5)$ & $13,29 \pm 5,92$ & $13(8)$ & & $1,39 \pm 2,88$ & $0(2)$ & & $27,28 \pm 3,87$ & $29(6)$ & & $22,79 \pm 8,54$ & $21(11)$ & \\
\hline \multicolumn{14}{|l|}{ Pareja actual } \\
\hline Sí & $49(20)$ & $13,37 \pm 5,77$ & $13(7)$ & 0,927 & $1,35 \pm 2,69$ & $0(2)$ & 0,324 & $29,22 \pm 4,08$ & $30(4)$ & 0,011 & $21,49 \pm 7,54$ & $21(11)$ & 0,354 \\
\hline No & $196(80)$ & $13,58 \pm 6,25$ & 13(9) & & $1,59 \pm 3,12$ & $0(2)$ & & $27,55 \pm 4,25$ & $28(6)$ & & $23,62 \pm 9,7$ & $22(12)$ & \\
\hline \multicolumn{14}{|l|}{ Lugar de procedencia } \\
\hline Lima & $184(75,1)$ & $13,6 \pm 5,81$ & $13(8)$ & 0,705 & $1,45 \pm 2,75$ & $0(2)$ & 0,9 & $27,74 \pm 4,33$ & $28(6)$ & 0,579 & $23,31 \pm 9,63$ & $21,5(12)$ & 0,791 \\
\hline Provincia & $58(23,7)$ & $13,55 \pm 7,18$ & $13(10)$ & & $1,84 \pm 3,85$ & $0(3)$ & & $28,38 \pm 4,03$ & $29(5)$ & & $23,02 \pm 8,44$ & $22(12)$ & \\
\hline \multicolumn{14}{|l|}{ Religión } \\
\hline Sí & $186(75,9)$ & $13,33 \pm 5,98$ & $12,5(8)$ & 0,499 & $1,46 \pm 3,11$ & $0(2)$ & 0,161 & $28,1 \pm 4,2$ & $29(5)$ & 0,12 & $22,69 \pm 9$ & 21(11) & 0,238 \\
\hline No & $59(24,1)$ & $14,17 \pm 6,65$ & $13(9)$ & & $1,8 \pm 2,79$ & $1(3)$ & & $27,19 \pm 4,41$ & $27(6)$ & & $24,78 \pm 10,23$ & $23(16)$ & \\
\hline \multicolumn{14}{|l|}{ ¿Ha reprobado algún curso? } \\
\hline Sí & $32(13,1)$ & $14,31 \pm 6,01$ & $13(8)$ & 0,452 & $2,91 \pm 4,9$ & $0,5(5)$ & 0,127 & $26,78 \pm 5,77$ & $29(7)$ & 0,564 & $26,44 \pm 12,34$ & & \\
\hline No & $213(86,9)$ & $13,42 \pm 6,17$ & $13(8)$ & & $1,33 \pm 2,57$ & $0(2)$ & & $28,05 \pm 3,98$ & $28(5)$ & & $22,7 \pm 8,72$ & & \\
\hline \multicolumn{14}{|l|}{ ¿Ha reprobado algún curso? } \\
\hline Sí & $32(13,1)$ & $14,31 \pm 6,01$ & $13(8)$ & 0,452 & $2,91 \pm 4,9$ & $0,5(5)$ & 0,127 & $26,78 \pm 5,77$ & $29(7)$ & 0,564 & $26,44 \pm 12,34$ & 23(15) & 0,195 \\
\hline No & $213(86,9)$ & $13,42 \pm 6,17$ & $13(8)$ & & $1,33 \pm 2,57$ & $0(2)$ & & $28,05 \pm 3,98$ & $28(5)$ & & $22,7 \pm 8,72$ & $21(13)$ & \\
\hline \multicolumn{14}{|l|}{ Especialidad que desea seguir } \\
\hline Centrada en el paciente & $198(80,8)$ & $13,86 \pm 6,26$ & $13(8)$ & 0,234 & $1,5 \pm 2,94$ & $0(2)$ & 0,679 & $28,03 \pm 4,24$ & $29(6)$ & 0,207 & $23,33 \pm 9,11$ & 22(12) & 0,499 \\
\hline Centrada en la tecnología & $39(15,9)$ & $12,18 \pm 4,88$ & $13(8)$ & & $1,13 \pm 2,07$ & $0(2)$ & & $27,38 \pm 3,52$ & $28(4)$ & & $22,10 \pm 7,31$ & 22(9) & \\
\hline
\end{tabular}

AE: Agotamiento emocional; Cl: Cinismo; EA: Eficacia Académica; MBI-SS: Maslach Burnout Inventory-Student Survey Valores p obtenidos a través de la prueba $\mathrm{U}$ de Mann-Whitney 
Tabla 4. Coeficientes de correlación entre la edad, horas de actividad universitaria, y horas de actividad recreativa, con las dimensiones de la JSPE-S y de la MBI-SS.

\begin{tabular}{|c|c|c|c|c|c|c|c|c|c|c|c|}
\hline & Edad & $\begin{array}{l}\text { Actividad } \\
\text { universitaria } \\
\text { (horas) }\end{array}$ & $\begin{array}{l}\text { Actividad } \\
\text { recreativa } \\
\text { (horas) }\end{array}$ & TP & CC & PP & $\begin{array}{l}\text { Total } \\
\text { JSPE }\end{array}$ & $\mathrm{AE}$ & Cl & EA & $\begin{array}{c}\text { Total } \\
\text { MBI-SS }\end{array}$ \\
\hline Edad & 1,000 & & & & & & & & & & \\
\hline $\begin{array}{l}\text { Actividad universitaria } \\
\text { (horas) }\end{array}$ & ,036 & 1,000 & & & & & & & & & \\
\hline Actividad recreativa (horas) &,- 057 &, 031 & 1,000 & & & & & & & & \\
\hline TP &,- 029 & ,034 &,- 027 & 1,000 & & & & & & & \\
\hline CC &,- 032 & 085 &,- 028 &, $452^{* *}$ & 1,000 & & & & & & \\
\hline PP &, 029 &,- 013 &,- 042 &, $215^{* *}$ &, $261^{* *}$ & 1,000 & & & & & \\
\hline Total JSPE &,- 022 &, 044 &,- 038 &, $850^{* *}$ &, $802^{* *}$ &, $457^{* *}$ & 1,000 & & & & \\
\hline$A E$ &,- 059 & 047 &,- 105 &,- 103 &,- 106 &,- 098 &,$- 137^{*}$ & 1,000 & & & \\
\hline $\mathrm{Cl}$ & ,041 &,$- 190^{* *}$ & ,062 &,$- 211^{* *}$ &,$- 194^{* *}$ & -101 &,$- 241^{* *}$ &, $327^{* *}$ & 1,000 & & \\
\hline EA &,- 061 &, $167^{* *}$ &,- 016 &, $291^{* *}$ &, $150^{*}$ &, $147^{*}$ &, $262^{* *}$ &,- 025 &,$- 211^{* *}$ & 1,000 & \\
\hline Total MBI-SS &,- 021 &,- 084 &,- 073 &,$- 245^{* *}$ &,$- 198^{* *}$ &,$- 163^{*}$ &,$- 269^{* *}$ &, $819^{* *}$ &, $539^{* *}$ &,$- 504^{* *}$ & 1,000 \\
\hline
\end{tabular}

$* * p<0,01$

$* p<0,05$

TP: Toma de perspectiva; CC: Cuidado con compasión; PP: Ponerse en los zapatos del paciente; JSPE-S: Jefferson Scale of Physician Empathy versión para estudiantes de medicina; AE: Agotamiento emocional; Cl: Cinismo; EA: Eficacia Académica; MBI-SS: Maslach Burnout Inventory-Student Survey

Valores $\mathrm{p}$ obtenidos a través de la correlación de Spearman

res puntuaciones de empatía, siendo esto probablemente debido a que el tener una pareja influye positivamente en el desarrollo emocional (25).

Encontramos que la empatía médica se correlacionó con todas las dimensiones del burnout; de forma negativa con el $\mathrm{AE}$ y $\mathrm{Cl}$, y de forma positiva con la EA. Estos resultados son similares a los reportados en otros estudios ${ }^{(2,26-28)}$. Aunque probablemente mayores niveles de burnout impliquen una disminución de la empatía, el mecanismo de acción podría ser en dirección opuesta, o incluso "circular" (28).

Debemos resaltar que, si bien las asociaciones reportadas fueron significativas, estas fueron de poca magnitud. En futuros estudios se deberá explorar otros factores importantes que pudieran influir en la empatía y el burnout, como los aspectos culturales y sociales del entorno de aprendizaje, las experiencias de los estudiantes en su institución académica, el plan de estudios, las interacciones con sus compañeros, profesores y autoridades universitarias, entre otras ${ }^{(9)}$, con la finalidad de explorar estas variables como moderadores de la relación entre ambos constructos.

Este estudio presenta limitaciones; en primer lugar, los resultados no pueden ser generalizables ya que estudiamos a una sola institución universitaria. Sin embargo, esta limitación podría ser menor si comprendemos que la FMH-USMP es similar a otras universidades privadas del Perú. Por otro lado, al tener un diseño transversal solo establecimos relación, más no causalidad. Finalmente, la JSPE-S y la MBI-SS, al ser escalas autoadministradas, podrían generar un sesgo en sus respuestas debido a la autocrítica hacia las actitudes poco empáticas o cínicas.

En conclusión, en esta muestra de estudiantes de medicina de primer año encontramos que la empatía médica muestra una correlación con todas las dimensiones del burnout; de forma negativa con el $\mathrm{AE} \mathrm{y} \mathrm{Cl}$, y de forma positiva con la EA. Además, encontramos una alta prevalencia de agotamiento emocional. Con estos resultados queremos resaltar la necesidad de que en las facultades de medicina se incluyan programas de prevención, detección y manejo de diversos problemas de salud mental, como el manejo del estrés.

\section{REFERENCIAS BIBLIOGRÁFICAS}

1. Yuguero O, Ramon Marsal J, Esquerda M, Vivanco L, Soler-Gonzalez J. Association bet- ween low empathy and high burnout among primary care physicians and nurses in Lleida, Spain. Eur J Gen Pract. 2017;23(1):4-10. DOI: 10.1080/13814788.2016.1233173

2. Chae SJ, Jeong SM, Chung YS. The mediating effect of calling on the relationship between medical school students' academic burnout and empathy. Korean J Med Educ. 2017;29(3):165-73. DOI: 10.3946/kjme.2017.62

3. Baron-Cohen S. Empatía cero. Nueva teoría de la crueldad. Madrid: Alianza Editorial; 2012. 229 p.

4. Halpern J. What is clinical empathy? J Gen Intern Med. 2003;18(8):670-4. DOI: 10.1046/j.15251497.2003.21017.x

5. Zenasni F, Boujut E, Woerner A, Sultan S. Burnout and empathy in primary care: three hypotheses. $\mathrm{Br}$ J Gen Pract. 2012;62(600):346-7. DOI: 10.3399/ bjgp12X652193

6. Worly B, Verbeck N, Walker C, Clinchot DM. Burnout, perceived stress, and empathic concern: differences in female and male Millennial medical students. Psychol Health Med. 2018:1-10. DOI:10.1080/1354 8506.2018.1529329

7. Maslach C, Leiter M, Schaufeli W. Measuring Burnout. In: Cartwright S, Cooper CL, Maslach C, Leiter MP, Schaufeli W, editors. The Oxford handbook of organizational well-being. Oxford: Oxford University Press; 2008. p. 86-108.

8. Boni R, Paiva CE, de Oliveira MA, Lucchetti G, Fregnani J, Paiva BSR. Burnout among medical students during the first years of undergraduate school: Prevalence and associated factors. PLoS One. 2018;13(3):e0191746. DOI: 10.1371/journal. pone. 0191746

9. Lucchetti G, Damiano RF, DiLalla LF, Lucchetti ALG, Moutinho ILD, da Silva Ezequiel O, et al. Crosscultural Differences in Mental Health, Quality of Life, Empathy, and Burnout between US and Brazilian Medical Students. Acad Psychiatry. 2018;42(1):627. DOI: $10.1007 /$ s40596-017-0777-2 
10. Thirioux B, Birault F, Jaafari N. Empathy Is a Protective Factor of Burnout in Physicians: New NeuroPhenomenological Hypotheses Regarding Empathy and Sympathy in Care Relationship. Front Psychol. 2016;7:763. DOI: 10.3389/fpsyg.2016.00763

11. Ñahui J. Asociación entre el Sindrome de Burnout y la Empatia en internos de ciencias de la salud del Hospital de Vitarte durante el mes de noviembre del año 2016 [Tesis para optar el grado de Médico Cirujano]. Lima: Universidad Ricardo Palma; 2017.

12. Hojat M, Gonnella JS, Nasca TJ, Mangione S, Veloksi JJ, Magee M. The Jefferson Scale of Physician Empathy: further psychometric data and differences by gender and specialty at item level. Acad Med. 2002;77(10 Suppl):S58-60. DOI: DOI: 10.1097/00001888-200210001-00019

13. Hojat M, Mangione S, Nasca T, Cohen M, Gonnella J, Erdmann J, et al. The Jefferson Scale of Physician Empathy: Development and Preliminary Psychometric Data. Educ Psychol Meas. 2001;61(2):349-65. DOI: https://doi.org/10.1177/00131640121971158

14. Alcorta A, González J, Tavitas S, Rodríguez F, Hojat M. Validación de la Escala de Empatía Médica de Jefferson en estudiantes de medicina mexicanos. Salud Ment (Méx). 2005;28(5):57-63.

15. Huarcaya-Victoria J, Rivera-Encinas MT. Evaluación de la empatía en alumnos de medicina humana de una universidad privada del Perú. Educ Med. 2019;20(Suppl 2):100-7. DOI: 10.1016/j.edumed.2018.05.008
16. Huarcaya-Victoria J, Cano-Uria B, Villanueva-Ruska A, de la Cruz-Oré J. Evaluación de los niveles de empatía médica en médicos residentes de un hospital general en Perú. Educ Med. 2019;20(Suppl 2):59-66. DOI: 10.1016/j.edumed.2018.04.004

17. Schaufeli WB, Martínez IM, Pinto AM, Salanova $M$, Bakker AB. Burnout and Engagement in University Students:A Cross-National Study. J Cross-Cult Psychol. 2002;33(5):464-81. DOI: $10.1177 / 0022022102033005003$

18. Hederich-Martínez C, Caballero-Domínguez CC. Validación del cuestionario Maslach Burnout InventoryStudent Survey (MBI-SS) en contexto académico colombiano. CES Psicologia. 2016;9(1):1-15.

19. Wachholtz A, Rogoff M. The relationship between spirituality and burnout among medical students. $J$ Contemp Med Educ. 2013;1(2):83-91. DOI: 10.5455/ jcme.20130104060612

20. Mazurkiewicz R, Korenstein D, Fallar R, Ripp J. The prevalence and correlations of medical student burnout in the pre-clinical years: a cross-sectional study. Psychol Health Med. 2012;17(2):188-95. DOI: 10.1080/13548506.2011.597770

21. Cecil J, McHale C, Hart J, Laidlaw A. Behaviou and burnout in medical students. Med Educ Online. 2014;19:25209. DOI: 10.3402/meo.v19.25209

22. Dyrbye LN, West CP, Satele D, Boone S, Tan L, Sloan J, et al. Burnout among U.S. medical students, residents, and early career physicians relative to the general U.S. population. Acad Med. 2014;89(3):44351. DOI: $10.1097 / \mathrm{acm} .0000000000000134$
23. Paro HB, Silveira PS, Perotta B, Gannam S, Enns SC, Giaxa RR, et al. Empathy among medical students: is there a relation with quality of life and burnout? PLoS One. 2014;9(4):e94133. DOI: 10.1371/journal.pone.0094133

24. Thomas MR, Dyrbye LN, Huntington JL, Lawson KL, Novotny PJ, Sloan JA, et al. How do distress and well-being relate to medical student empathy? A multicenter study. J Gen Intern Med. 2007;22(2):177-83. DOI: 10.1007/s11606-006-0039-6

25. Park C, Lee YJ, Hong M, Jung $C H$, Synn $Y$, Kwack YS, et al. A Multicenter Study Investigating Empathy and Burnout Characteristics in Medical Residents with Various Specialties. J Korean Med Sci. 2016;31(4):590-7. DOI: 10.3346/jkms.2016.31.4.590

26. Hojat M, Vergare M, Isenberg $G$, Cohen M, Spandorfer J. Underlying construct of empathy, optimism, and burnout in medical students. Int J Med Educ. 2015;6:12-6. DOI: 10.5116/ijme.54c3.60cd

27. von Harscher $H$, Desmarais $N$, Dollinger $R$, Grossman S, Aldana S. The impact of empathy on burnout in medical students: new findings. Psychol Health Med. 2018;23(3):295-303. DOI: 10.1080/13548506.2017.1374545

28. Yuguero O, Forne C, Esquerda M, Pifarre J, Abadias MJ, Vinas J. Empathy and burnout of emergency professionals of a health region: A cross-sectional study. Medicine (Baltimore). 2017;96(37):e8030. DOI: $10.1097 / \mathrm{md} .0000000000008030$ 